\title{
The molecular mechanisms that control thrombopoiesis
}

\author{
Kenneth Kaushansky \\ Department of Medicine, Division of Hematology/Oncology, University of California, San Diego, San Diego, California, USA.
}

\begin{abstract}
Our understanding of thrombopoiesis - the formation of blood platelets - has improved greatly in the last decade, with the cloning and characterization of thrombopoietin, the primary regulator of this process. Thrombopoietin affects nearly all aspects of platelet production, from self-renewal and expansion of HSCs, through stimulation of the proliferation of megakaryocyte progenitor cells, to support of the maturation of these cells into platelet-producing cells. The molecular and cellular mechanisms through which thrombopoietin affects platelet production provide new insights into the interplay between intrinsic and extrinsic influences on hematopoiesis and highlight new opportunities to translate basic biology into clinical advances.
\end{abstract}

\section{Overview of platelets and thrombopoiesis}

An adequate supply of platelets is essential to repair the minute vascular damage that occurs with daily life, and to initiate thrombus formation in the event of overt vascular injury. Accumulating evidence also indicates vital roles for platelets in wound repair, the innate immune response, and metastatic tumor cell biology. The average platelet count in humans ranges from $150 \times 10^{9}$ to $400 \times 10^{9}$ per liter, although the level for any individual is maintained within fairly narrow limits from day to day. While $150 \times 10^{9}$ to $400 \times 10^{9}$ per liter is considered "normal," the values derived from the mean \pm 2 SDs of a group of "healthy" individuals, epidemiological evidence indicates that individuals who display platelet counts in the highest quartile of the normal range have a 2 -fold increased risk of adverse cardiovascular events (1), and, in both experimental animal models of metastatic cancer and patients with tumors, higher platelet levels carry an unfavorable prognosis (2).

With a lifespan of approximately 10 days, a blood volume of 5 liters, and one-third of platelets pooled in the spleen, the average adult must produce each day approximately $1 \times 10^{11}$ platelets to maintain a normal platelet count under steady-state conditions, a level of production that can increase more than 10-fold under conditions of increased demand. The primary regulator of platelet production is thrombopoietin, an acidic glycoprotein produced primarily in the liver, kidney, and BM. The biochemistry and structure-activity relationships of thrombopoietin have been carefully evaluated, as have the binding sites to its receptor, the product of the cellular protooncogene $c-\operatorname{Mpl}(3,4)$. This Review will focus on the regulation of platelet production, how thrombopoietin stimulates thrombopoiesis under normal and pathological conditions, and the molecular mechanisms through which the hormone produces its biological effects. In addition to providing an understanding of normal physiology, the discovery of the biological effects of thrombopoietin and its receptor provides a platform on which to understand a number of clinical disorders of hematopoiesis and serves to suggest novel therapeutic approaches to several diseases.

Nonstandard abbreviations used: ET, essential thrombocythemia; GSK3 $\beta$, glycogen synthase kinase-3 $\beta$; IMF, idiopathic myelofibrosis; ORF, open reading frame; PV, polycythemia vera; SDF-1, stromal cell-derived factor-1.

Conflict of interest: The author has declared that no conflict of interest exists.

Citation for this article: J. Clin. Invest. 115:3339-3347 (2005).

doi:10.1172/JCI26674.

\section{Discovery of thrombopoietin and the c-Mpl receptor}

Evidence for a humoral regulator of thrombopoiesis was first provided in the late 1950s when plasma from bleeding or thrombocytopenic rats was found to induce thrombocytosis when transfused into secondary animals. Based on ongoing work in erythropoiesis, the term "thrombopoietin" was first coined in 1958 to describe the humoral substance responsible for increasing platelet production (5). In the late 1960s, in vivo assays were developed to detect thrombopoietin (6) but were cumbersome, hampering its purification. In the 1980s, in vitro megakaryocyte colony-forming assays were developed, allowing the identification of megakaryocyte colonystimulating factors, but whether the identified substances, such as IL-3, GM-CSF, IL-6, or IL-11, were the same as thrombopoietin remained controversial (7).

Occasionally in science, findings from one discipline spur an entirely distinct field of investigation. The description of the murine myeloproliferative leukemia virus in 1986 (8) and its corresponding oncogene $(\nu-M p l)$ and cellular protooncogene $(c-M p l)$ in the early 1990 s $(9,10)$ had such an effect on the search for thrombopoietin. Based on a number of shared primary and secondary structural features, $\mathrm{c}-\mathrm{Mpl}$ was identified as an orphan member of the hematopoietic cytokine receptor family of proteins, leading to the cloning of its ligand, thrombopoietin, 2 years later (reviewed in ref. 11). Initial experiments with the recombinant protein established thrombopoietin as the primary physiological regulator of thrombopoiesis, as its levels were inversely related to platelet or megakaryocyte mass and its infusion massively increased platelet production (12).

\section{Regulation of thrombopoietin production}

Investigators have found that blood and marrow levels of thrombopoietin are inversely related to platelet count. Patients with aplastic anemia or thrombocytopenia secondary to myelosuppressive therapy display high levels of the hormone $(13,14)$. However, there are some notable exceptions to this relationship. The first is seen in states of platelet destruction, where levels of the hormone are not as high as would be anticipated from the degree of thrombocytopenia $(15,16)$. Such instances, seen in most patients with idiopathic thrombocytopenic purpura, are characterized by megakaryocyte hypertrophy, which likely contributes to thrombopoietin regulation (17). A second instance in which thrombopoietin levels are not accurately predicted by blood platelet count is in patients with inflam- 


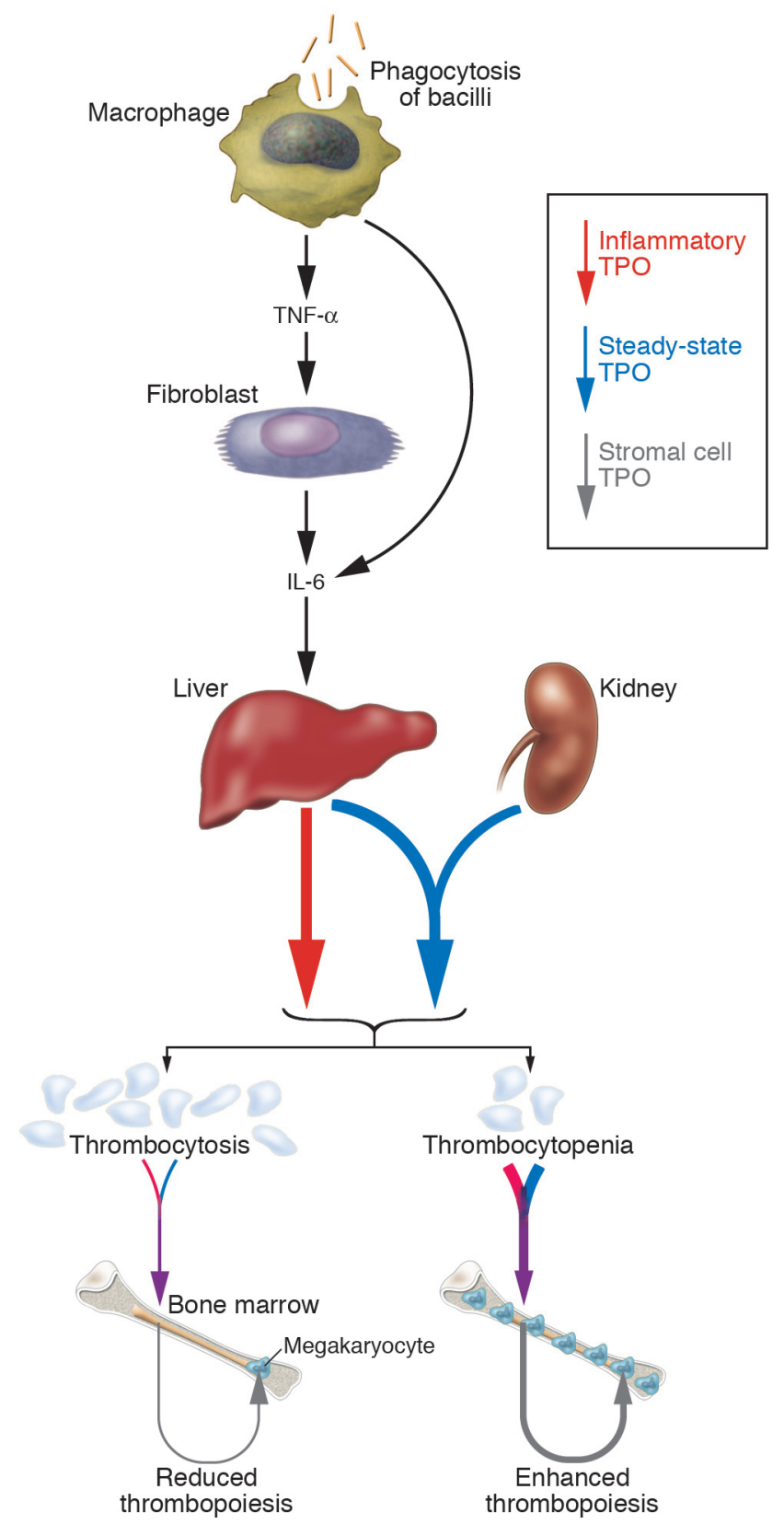

matory, reactive thrombocytosis, where levels of the hormone are higher than expected (18-21). However, an important unanswered question is whether alterations in thrombopoietin production explain the thrombocytosis associated with iron deficiency.

A major component of thrombopoietin regulation is achieved by receptor-mediated uptake and destruction (Figure 1), a mechanism of hematopoietic growth factor regulation first established for M-CSF (22). Platelets bear high-affinity thrombopoietin receptors that remove the hormone from solution (23), thereby establishing an autoregulatory loop; as platelet counts rise, they remove more of the hormone from the circulation, driving levels down, whereas in thrombocytopenic states there are less platelets to adsorb thrombopoietin, allowing levels to rise and drive increased thrombopoiesis. However, not all thrombopoietin receptors contribute to this effect; while endothelial cells display c-Mpl receptors (24) and some

\section{Figure 1}

The regulation of thrombopoietin levels. A steady-state amount of hepatic thrombopoietin (TPO) is regulated by platelet c-Mpl receptormediated uptake and destruction of the hormone. Hepatic production of the hormone is depicted. Upon binding to platelet c-Mpl receptors, the hormone is removed from the circulation and destroyed, which reduces blood levels. In the presence of inflammation, IL-6 is released from macrophages and, through TNF- $\alpha$ stimulation, from fibroblasts and circulates to the liver to enhance thrombopoietin production. Thrombocytopenia also leads to enhanced marrow stromal cell production of thrombopoietin, although the molecular mediator(s) of this effect is not yet completely understood.

endothelial cell types proliferate or migrate in response to the hormone (25), transplantation studies have shown that endothelial cell c-Mpl does not materially affect thrombopoietin levels despite a 100 -fold more expansive cell surface (and predicted greater c-Mpl mass) than that displayed by the totality of megakaryocytes and platelets (26). Therefore, the mere presence of c-Mpl does not guarantee that it is involved in regulating thrombopoietin blood levels.

A second exception to the relatively simple platelet-adsorptionand-destruction model of platelet homeostasis is illustrated by the physiological response to severe thrombocytopenia; studies in both mice and humans show that while marrow stromal cells display very little thrombopoietin mRNA under normal conditions, transcripts for the cytokine greatly increase in the presence of thrombocytopenia (Figure 1) (27). The precise humoral or cellular mediators of this effect are under intense study. For example, in one report, the platelet $\alpha$-granule proteins PDGF and FGF- 2 increased, but platelet factor 4, thrombospondin, and TGF- $\beta$ decreased thrombopoietin production from primary human BM stromal cells (28). However, others have reported that HGF is responsible for thrombopoietin production from hepatocytes (29).

A third mechanism of thrombopoietin regulation occurs in states of reactive thrombocytosis, where hormone concentrations are higher than that predicted by the degree of thrombocytosis. For example, inflammatory stimuli affect thrombopoietin production, with the acute-phase response mediator IL- 6 increasing thrombopoietin transcription from the liver (30). These in vitro effects are also seen in vivo; administration of IL- 6 to mice or cancer patients increases thrombopoietin-specific mRNA in the liver and levels of the hormone in the blood. Since an anti-thrombopoietin antibody neutralizes the thrombopoietic effects of administered IL-6 (30), it is now clear that thrombopoietin is the final mediator of inflammation-induced thrombocytosis.

\section{Additional modes of thrombopoietic regulation}

In addition to thrombopoietin, additional factors likely influence thrombopoiesis, as the genetic elimination of thrombopoietin or its receptor leads to profound but not absolute thrombocytopenia (the platelet counts in these settings are about $10 \%$ of a normal level). In order to determine whether any of the known hematopoietic cytokines contributes to the residual thrombopoiesis in the $c-M p l$-null state, such mice have been crossed with other cytokine- or cytokine receptor-deficient animals; from these studies it is clear that IL-3 $(31,32)$, IL-6, IL-11, and LIF (33) are not basal, physiological mediators of thrombopoiesis. However, the chemokine stromal cell-derived factor-1 (SDF-1) exerts numerous influences on megakaryopoiesis, and studies indicate that it may be responsible for thrombopoiesis not related to thrombopoietin. 
For example, SDF-1 acts alone and in synergy with thrombopoietin to enhance megakaryocyte colony formation in serum-free culture (34). The chemokine also affects the motility of megakaryocytes, driving their migration toward stromal cells (35), with which they productively interact in an integrin $\alpha_{4} \beta_{1}$-dependent manner (36). The administration of SDF-1, along with FGF-4, can nearly normalize the platelet count of c-Mpl-deficient mice and can enhance platelet recovery following myelosuppression (37). Thus, accumulating evidence points to SDF-1 and marrow stromal cells as important influences on thrombopoiesis, but whether levels of the chemokine or surface expression of integrins can be modulated in response to thrombocytopenia remains unknown.

\section{Hematopoietic and other activities of thrombopoietin}

Although thrombopoietin was initially postulated to exclusively promote the maturation of megakaryocytes and their fragmentation into platelets, its biological effects are more wide ranging than was initially thought. Thrombopoietin supports the survival and expansion of HSCs and all types of progenitor cells that display megakaryocyte potential, promotes the maturation of megakaryocytes, and enhances the platelet response to activating events.

Thrombopoietin is the most potent single stimulus of the growth of hematopoietic progenitor cells committed to the megakaryocyte lineage. It also acts in synergy with other hematopoietic cytokines, including SCF, IL-11, and erythropoietin, to promote progenitor cell proliferation (38). In suspension culture, thrombopoietin stimulates the formation of large, highly polyploid megakaryocytes that can form proplatelet processes that then fragment into immature and mature platelets (39). The hormone also affects mature platelets, reducing the level of ADP, collagen, or thrombin needed to induce aggregation $(40,41)$, and enhances platelet adhesion to fibrinogen, fibronectin, and vWF in parallel plate perfusion chamber assays (42).

In addition to its stimulation of most, if not all, aspects of megakaryopoiesis, thrombopoietin displays profound and nonredundant effects on HSCs. Using highly purified marrow-derived cells, 2 groups showed that, by itself, the hormone affects the survival of HSCs and works in synergy with IL-3 or SCF to promote proliferation in vitro in both murine and human cells $(43,44)$. Subsequent in vivo studies confirmed the physiological relevance of these findings; genetic elimination of thrombopoietin or its receptor in mice, or congenital absence of c-Mpl in children, leads to profound thrombocytopenia, and to equivalent reductions in the levels of HSCs and progenitor cells of all hematopoietic lineages (45-48).

While thrombopoietin clearly plays an important role in the maintenance of HSC numbers, it is not responsible for determination of the lineage fate of these cells. The distribution of hematopoietic progenitor cell types that emerge from cultures of HSCs is identical whether thrombopoietin is present or not (43). Rather, it is likely that the balance of transcription factors present in the HSC is responsible for commitment to one or another hematopoietic lineage. Recently, based on commitment to either the erythroid or the myeloid lineage, a model of mutual transcription factor antagonism has developed that can explain much of the stochastic commitment to one or another cell lineage $(49,50)$. For megakaryocytes, $c-M y b$ seems particularly important; hypomorphic alleles of the gene result in megakaryocytic expansion (51), and 2 mutant $c-M y b$ alleles, identified in a chemical mutagenesis screen, have been shown to skew lineage commitment toward megakaryopoiesis and alter cell responsiveness to hematopoietic growth fac- tors (52). The transcription factors known to affect megakaryocyte commitment have been recently reviewed (53) and include GATA1, AML1/RUNX1, FOG1, FLI1, MYB, and NF-E2.

\section{Mpl receptor expression, regulation, and signaling}

The type I hematopoietic growth factor receptor family, of which $\mathrm{c}-\mathrm{Mpl}$ is a member, consists of more than 20 molecules that bear 1 or 2 cytokine receptor motifs, an approximately 200-amino acid module containing 4 spatially conserved Cys residues, $14 \beta$-sheets, and a juxtamembrane Trp-Ser-Xaa-Trp-Ser sequence (54). In addition to the cytokine receptor motif(s), type I receptors contain a 20- to 25-residue transmembrane domain and a 70- to 500-amino acid intracellular domain containing short sequences that bind intracellular kinases and other signal-transducing molecules. The thrombopoietin receptor is expressed primarily in hematopoietic tissues, specifically in megakaryocytes, their precursors, and their progeny. For the most part, $\mathrm{c}-\mathrm{Mpl}$ is constitutively expressed in these tissues, although receptor display is modulated by thrombopoietin binding and receptor internalization. A second potential level of c-Mpl regulation exists; multiple spliceoforms of the receptor have been described that vary in their biological activity, and 1 form can alter receptor catabolism. Although the proportion of the various isoforms of the receptor differs in different tissues, they have not yet been shown to exert a regulatory effect.

The $c-M p l$ gene contains 12 exons and is organized like other members of the hematopoietic cytokine receptor family (55). A site for initiation of $c-M p l$ transcription resides 13 nucleotides upstream of the translation initiation codon, and although the promoter lacks conventional TATA and CAAT motifs, the 5' flanking sequence contains consensus binding sequences for Ets and GATA transcription factors, proteins vital for the regulation of many megakaryocyte-specific genes. Analysis of $c-M p l$ transcripts has identified several alternately spliced forms, including extracellular domain deletions (56), an alternate intracellular domain (the $\mathrm{K}$ isoform; ref. 8), and a prematurely truncated isoform containing a unique carboxyl terminus (the Mpl-tr isoform; ref. 57). While potentially acting as a dominant-negative form of the receptor, and differentially expressed in certain cell types, the $\mathrm{K}$ isoform does not affect thrombopoietin signaling, as it does not interact with the wild-type receptor (58). However, Mpl-tr may play a physiological role, as it is the only isoform expressed in both human and murine cells. Of note, expression levels of c-Mpl are low, with only 25-100 surface receptors present per platelet $(59,60)$. The origin of the poor expression of $\mathrm{c}-\mathrm{Mpl}$ appears to be related to the c-Mpl-tr isoform, as its coexpression with full-length c-Mpl leads to rapid degradation of the latter (57). However, whether this physiology is reflected in thrombopoietin signal regulation is, at present, only speculative.

Another aspect of c-Mpl regulation under intense study is its expression on hematopoietic cells of patients with myeloproliferative disorders (MPDs). While easily detectable on normal marrow megakaryocytes and platelets, the receptor is decreased on cells from patients with polycythemia vera and other myeloproliferative diseases $(61,62)$. While the molecular basis for this is not understood, it could be related to the hypersensitivity to cytokines and signaling abnormalities seen in these disorders. Another clue to this finding may lie in 2 recent observations, that coexpression of the signaling kinase JAK2 is vital for hematopoietic cytokine receptor expression (63), and that the activity of this kinase is altered in a substantial number of patients with MPDs. 


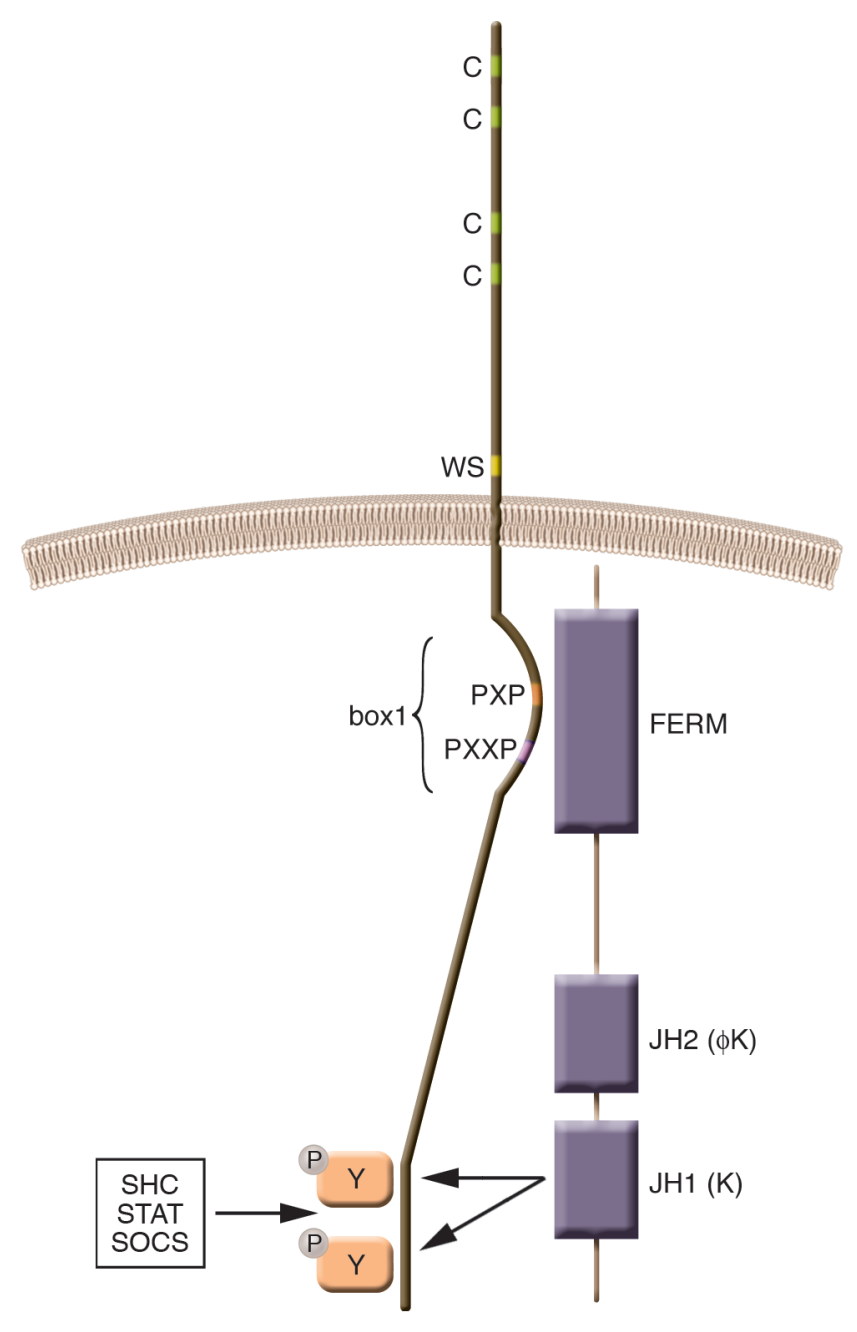

Upon binding, cognate ligand hematopoietic cytokine receptors such as c-Mpl are activated to transmit numerous biochemical signals. The molecular details of this process are now well understood based on studies of the erythropoietin receptor (EpoR). The EpoR exists in a homodimeric state in the absence of ligand, in a conformation that holds the cytoplasmic domains $73 \AA$ apart (64). Upon ligand binding, receptor conformation shifts, bringing the cytoplasmic domains within $39 \AA$ A of one another. Additional studies indicate that the membrane-proximal box 1 and box 2 cytoplasmic domains constitutively bind JAK family kinases, even in an inactive state. Upon ligand binding, the closer juxtaposition of the 2 tethered kinases allows their cross-activation, initiating signal transduction. The active JAK kinase then phosphorylates (a) tyrosine residues within the receptor itself; (b) molecules that promote cell survival and proliferation, including the STATs, PI3K, and the MAPKs; and (c) those that limit cell signaling, including the SHP1 and SHIP1 phosphatases and SOCSs (Figures 2 and 3).

Additional insights into how the JAK kinases are regulated come from domain analysis of the proteins. All 4 members of the family (JAK1, JAK2, JAK3, and TYK2) display 3 major domains, JH1 (JAK homology 1), JH2, and FERM (four-point-one, ezrin, radixin, moe$\sin$ ), the latter responsible for binding to the cytoplasmic domain of the cytokine receptors (Figure 2). The JH1 domain carries the kinase activity of JAKs, and while JH2 bears significant homology

\section{Figure 2}

Hematopoietic cytokine receptor architecture and mechanism of initial signaling. A stylized hematopoietic cytokine receptor is shown, depicting the 1 or 2 cytokine receptor motifs (C, Cys; WS, Trp-SerXaa-Trp-Ser), the transmembrane domain, and the box1 sequence to which JAK kinases bind. Also shown are the 3 major domains of JAK kinases, the FERM domain, which binds to box1, and the kinase $\mathrm{JH} 1$ and regulatory $\mathrm{JH} 2$ domains. Finally, upon JAK activation, the site of receptor tyrosine phosphorylation is shown, which then serves as a docking site for STATs and adapter proteins (SHC or SHP2).

to $\mathrm{JH} 1$, its active site is altered and inactivated and is thus termed the pseudokinase $\phi \mathrm{K}$ domain. The function of JH 2 was identified by differential expression studies; the JH1 domain is an active kinase when expressed alone, whereas the activity of a JH1/JH2 polypeptide is greatly blunted (65). Thus, the JH2 domain regulates the kinase activity of JH1, a physiology put into structural terms by homology modeling of $\mathrm{JH} 1 / \mathrm{JH} 2$; the $\mathrm{JH} 2$ domain interacts with the inactive, but not the active, conformation of the activation loop of JH1 (Figure 4; ref. 66), in a region of JH2 shown to be vital for kinase regulatory activity.

Once c-Mpl is activated by thrombopoietin engagement, its multiple effects on HSCs, megakaryocytes, and platelets are mediated by a series of biochemical signaling events. Thrombopoietin activates both JAK2 and TYK2 in c-Mpl-bearing cell lines, although only JAK2 is essential for signaling and is the predominant isoform activated in primary megakaryocytes (67). By generating a complex composed of the phosphatase SHP2, a scaffolding Gab/IRS protein, and the $\mathrm{p} 85$ regulatory subunit of $\mathrm{PI} 3 \mathrm{~K}$, thrombopoietin stimulation of megakaryocytes and their precursors activates PI3K and its immediate downstream effector Akt (PKB) (Figure 3) (68, 69). Blocking this pathway inhibits thrombopoietin-induced cell survival and proliferation (70). In the mature platelet, the hormone enhances $\alpha$-granule secretion and aggregation induced by thrombin in a PI3K-dependent fashion (71). The pathways downstream of Akt in megakaryocytes and platelets are under study and include the transcription factor FOXO3a, the cell cycle inhibitor p27, and glycogen synthase kinase- $3 \beta$ (GSK3 $\beta$ ). In addition to PI3K, thrombopoietin stimulates 2 of the MAPK pathways (Figure 3), p42/p44 ERK1 and ERK2 (72) and p38 MAPK (73), events mediated by receptor phosphorylation, binding and phosphorylation of Grb2, SHC, and SOS, and exchange of GDP for GTP on Ras (74). The functional consequences of these events include induction of the transcription factor HoxB4 and expansion of HSCs mediated by p38 MAPK (73); translocation of the transcription factor HoxA9 from cytoplasm to nucleus, which also favorably affects HSC expansion (75); the ERK1/2-induced proliferation and polyploidization of megakaryocytes (76); and augmented thrombin-induced liberation of phospholipase $\mathrm{A}_{2}$ and platelet activation (77).

\section{Implications of thrombopoietin/Mpl signaling in clinical disorders of hematopoiesis}

Two primary reasons to generate a detailed map of the signaling circuitry used by hematopoietic growth factors are to understand disorders of hematopoietic growth and to intervene in these processes for therapeutic benefit (either enhancing or blunting signaling). A number of human diseases in which blood cell production is altered can now be understood as disorders of growth factor signaling.

One of the most common abnormalities of the blood count is elevated platelet levels, and the most common cause of thrombo- 


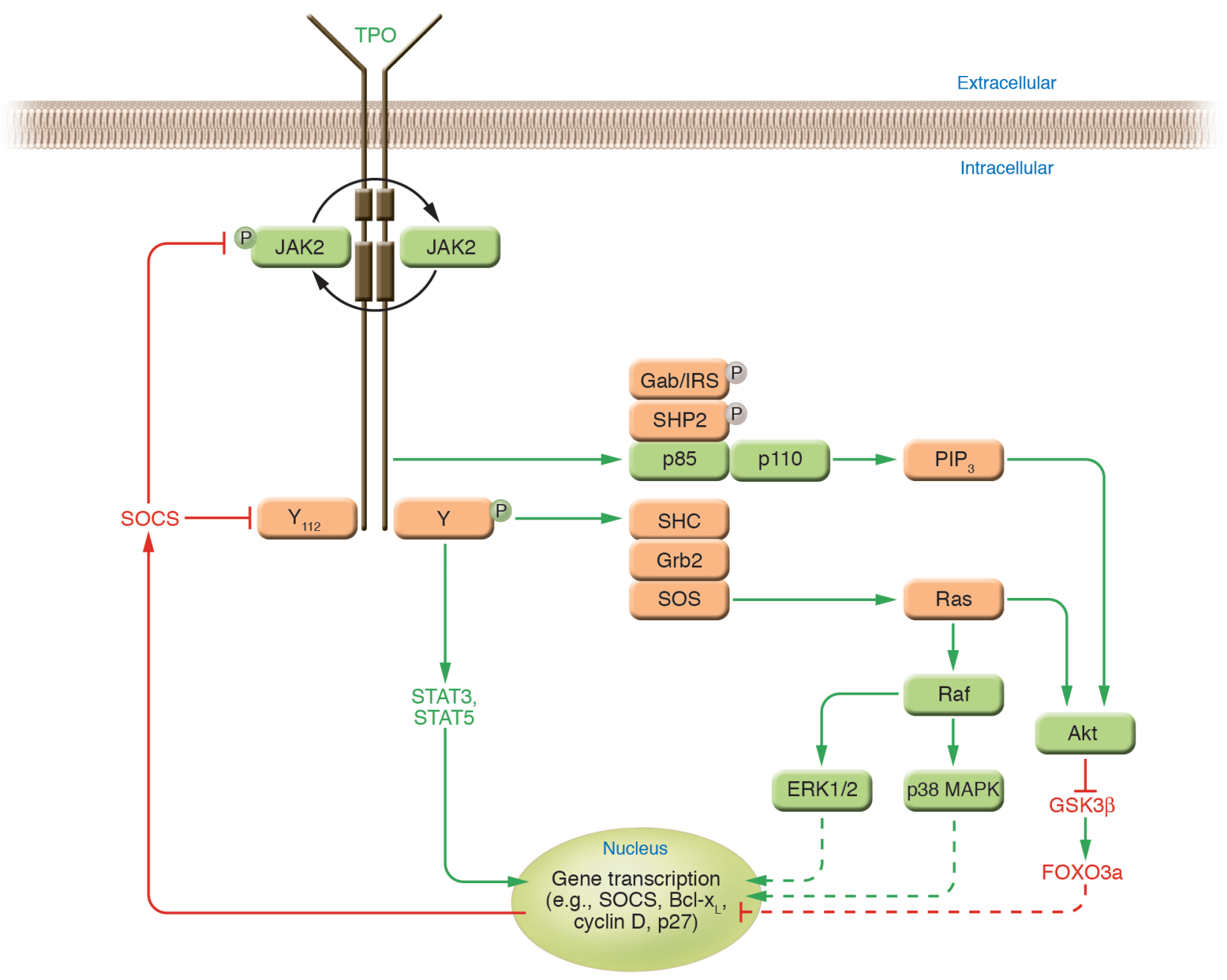

Figure 3

Signaling pathways activated by thrombopoietin. A stylized drawing of c-Mpl is shown in the activated (phosphorylated) form. Once phosphorylated, Tyr $_{112}$ serves as a docking site for STAT3 and STAT5, both activated by thrombopoietin in megakaryocytes, which leads to production of $\mathrm{Bcl}-\mathrm{x}_{\mathrm{L}}$, among other antiapoptotic and pro-proliferative signaling molecules. The same site also serves to recruit $\mathrm{SHC}$, which in turn recruits Grb2 and SOS (the latter a guanine nucleotide exchange factor for Ras), exchanging GTP for GDP, and thereby activating Ras. In succession, a MAPKKK (MAPK kinase kinase, e.g., Raf), a MAPKK (MAPK kinase), and the MAPK ERK1/2 or p38 MAPK are recruited and activated. As shown, Raf activation also contributes to PI3K activation. At a site proximal to Tyr ${ }_{112}$, a complex containing the phosphatase SHP2, the adapter protein Gab1, and the regulatory subunit of PI3K (p85) forms upon phosphorylation by JAK2, which recruits the kinase subunit of $\mathrm{PI3K}(\mathrm{p} 110)$, leading to phosphorylation of cell membrane-bound phosphoinositol ${ }_{4,5}$ biphosphate $\left(\mathrm{PIP}_{2}\right)$ and thus generating phosphoinositol ${ }_{3,4,5}$ triphosphate $\left(\mathrm{PIP}_{3}\right)$. $\mathrm{PIP}_{3}$ then recruits pleckstrin homology domain-containing proteins, including the Ser/Thr protein tyrosine kinase Akt. Once activated at the cell membrane, Akt phosphorylates (and inactivates) GSK3 $\beta$, which also promotes cell proliferation. Akt also phosphorylates the transcription factor FOXO3a, leading to its nuclear exit and thus precluding its induction of the cell cycle inhibitor p27. Inhibition of cell signaling is also initiated by JAK activation; shown in red is the transcriptional regulation of SOCS proteins by STATs, and their subsequent blockade of signaling by preclusion of signaling molecule docking to P-Tyr residues of the receptor or their JAK-induced phosphorylation.

cytosis is reaction to an inflammatory insult (20). A number of cytokines are released from activated monocytes and macrophages, including IL-1, TNF- $\alpha$, and IL-6. While IL- 1 and TNF- $\alpha$ have mixed effects on hematopoiesis, in vitro IL- 6 appears to act as a megakaryocyte maturation factor (78). Intravenous infusion of IL-6, in mice and during clinical trials in humans, leads to modest thrombocytosis $(79,80)$. While this initially suggested that inflammatory thrombocytosis is due to IL-6, several investigators have more recently shown that IL- 6 induces hepatic thrombopoietin production and that the thrombocytosis associated with IL- 6 is eliminated by blockade of thrombopoietin action (Figure 1) (30).
Much less common than inflammatory thrombocytosis is familial thrombocytosis. At least 4 different genetic alterations of the thrombopoietin gene have been shown to enhance production of the hormone and drive polyclonal thrombocytosis. The basis of all these cases is enhanced translation efficiency of the thrombopoietin transcript.

Unlike most structural genes, in which protein translation initiates from the first AUG codon in the transcript, the open reading frame (ORF) encoding thrombopoietin begins with the eighth AUG present in the mRNA (Figure 5); the previous 7 ORFs encode short, apparently functionless polypeptides, if they are translated 


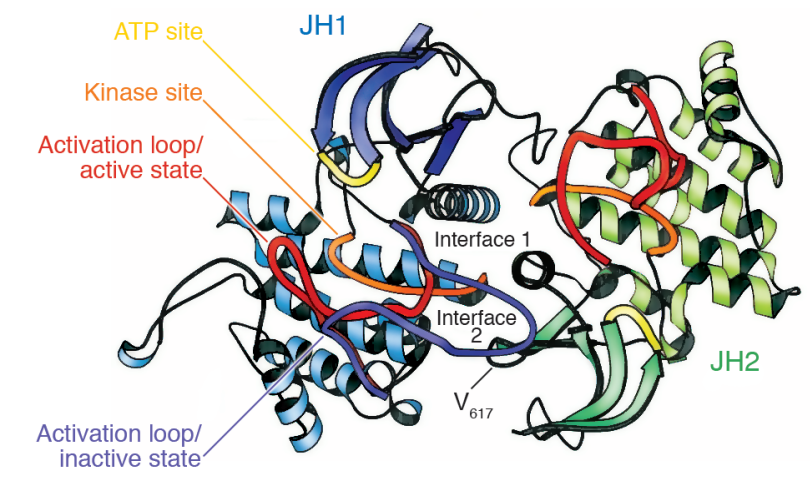

Figure 4

A molecular model of JAK2 JH1 and JH2 domains. Based on the tertiary structure of the dimer receptor tyrosine kinase FGF receptor-4, the model depicts the ATP-binding site (yellow), the kinase active site (orange), the activation loop of $\mathrm{JH} 1$ in both inactive (purple) and active (red) conformations, and the location of $\mathrm{JH} 2$ residue $\mathrm{Val}_{617}\left(\mathrm{~V}_{617}\right)$. Adapted with permission from Protein Engineering (66).

at all. Since the thrombopoietin initiation codon (AUG8) is embedded within the out-of-frame, seventh ORF, and since post-termination ribosomes cannot scan backward to initiate at upstream AUG codons (81), should a ribosome initiate at ORF7, it will terminate downstream of AUG8 and hence fail to translate thrombopoietin. Because of this alignment, thrombopoietin production is normally very inefficient. In 4 separate pedigrees displaying autosomal dominant thrombocytosis, the region surrounding the eighth AUG carries a single-nucleotide mutation that greatly enhances thrombopoietin translational efficiency (Figure 5; ref. 82). In 2 cases, a splice donor site mutation eliminates the exon carrying the seventh and eighth AUG codons, and the translation termination codon for the fifth and sixth ORFs, and results in an in-frame fusion of the fifth AUG to the thrombopoietin ORF. As the fifth AUG is a highly efficient initiation codon, thrombopoietin production rises, enhancing thrombopoiesis. The other 2 mutations within exon 3 of the thrombopoietin gene eliminate the reason for the unfavorable translation efficiency of the gene, the embedding of AUG8 within the seventh ORF (Figure 5), again resulting in greatly enhanced thrombopoietin production.

\section{Disorders of the c-Mpl receptor}

Thrombopoietin is the primary regulator of platelet production; thus, abnormalities of the hormone or its receptor might also be

\section{Figure 5}

Genetic alterations in thrombopoietin that lead to enhanced translation efficiency. The normal thrombopoietin mRNA (light blue) is spliced from 7 exons, of which 3 are shown (A). The numbered initiation codons found in the primary thrombopoietin transcript are shown as within their corresponding ORFs (e.g., the thrombopoietin ORF is dark blue and initiates from AUG8). The sites of mutation that lead to enhanced translation of the thrombopoietin transcript do so (B) by eliminating exon 3 by altered splicing $(\Delta \mathrm{E} 3)$ to create a new thrombopoietin ORF initiated by a highly efficient initiation codon (AUG5); (C) by nonsense mutation, prematurely truncating ORF7, which embeds the normal thrombopoietin ORF; or (D) by shifting the efficiently initiated ORF7 (by a single-nucleotide insertion) to now include the thrombopoietin polypeptide. Adapted with permission from Blood (82). responsible for thrombocytopenia. In numerous instances of congenital amegakaryocytic thrombocytopenia, either severe homozygous or mixed heterozygous, missense or nonsense mutations of the $c-M p l$ gene have been identified $(46,83,84)$. Loss of the receptor leads to severe congenital thrombocytopenia (platelet counts $\sim 20 \times 10^{9}$ per liter), and within $1-3$ years of birth nearly every patient harboring 2 severely mutant alleles develops aplastic anemia $(46,85)$, due to stem cell exhaustion. Stem cell transplantation is the only known treatment for the disease. Of note, while humans carrying inactivating mutations in the thrombopoietin receptor develop stem cell failure, despite a 10-fold reduction in stem cell numbers in both $c-M p l$-null and thrombopoietin-null mice, the animals maintain hematopoiesis and live a normal lifespan. The difference between mice and humans likely resides in different stem cell kinetics, although this issue has yet to be addressed.

The c-Mpl receptor was first recognized as a viral oncogene, in which most of the extracellular domain of the receptor was replaced by a viral gag gene sequence (7). It is likely that the uncontrolled myeloproliferation seen in mice expressing v-Mpl reflects the loss of the amino terminus of the receptor; simple truncation of the membrane-distal domain of c-Mpl eliminates its capacity to bind thrombopoietin, and its expression leads to thrombopoietin-independent growth of cells (86). This and other studies have spurred the concept that the membrane-distal domain puts a brake on constitutive signaling by the membrane-proximal and transmembrane domains, a block relieved by thrombopoietin binding. Several clinical observations are consistent with this model of c-Mpl activation. For example, an activating Ser-to-Asn mutation of the transmembrane domain of the $\mathrm{c}-\mathrm{Mpl}$ receptor, first recognized by random mutagenesis of the receptor (87), has now been found to cause thrombocytosis in a large family (88). A second disorder of the c-Mpl receptor associated with thrombocytosis has been found in African Americans, termed $\mathrm{Mpl}_{\text {Baltimore, a }}$ single-nucleotide polymorphism causing an amino acid alteration at position $\mathrm{Asn}_{34}$ of the receptor (89). While this alteration in the membrane-distal extracellular domain of c-Mpl associated with thrombocytosis also supports the developing model of c-Mpl activation, it remains possible that $\mathrm{Mpl}_{\text {Baltimore }}$ leads to thrombocytosis through another mechanism, or that the single-nucleotide poly-

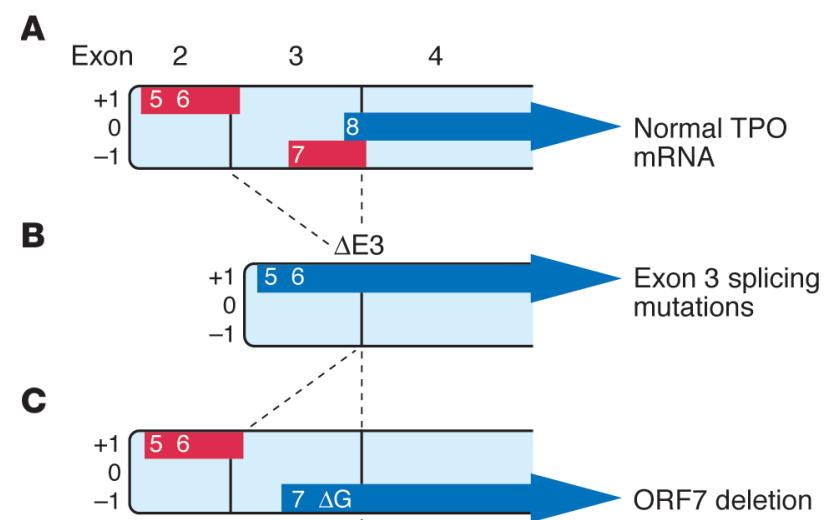

D

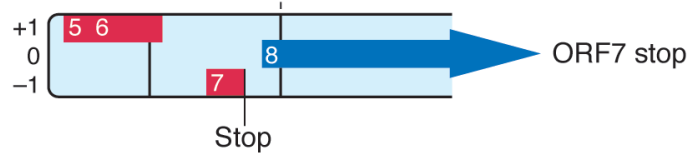


morphism lies in linkage disequilibrium with a distinct, causative, non-c-Mpl coding mutation in these individuals.

\section{Disorders of hematopoietic cell signal transduction}

The classic chronic MPDs polycythemia vera (PV), idiopathic myelofibrosis (IMF), and essential thrombocythemia (ET) share several clinical features, including overproduction of 1 or more hematopoietic cell lineages, a propensity for pathological hemorrhage or thrombosis, an excess or abnormality of megakaryocytes, which elaborate cytokines responsible for myelofibrosis, and a modestly elevated risk of progression to leukemia, a risk greatly hastened by exposure to alkylating agents (90). Several molecular features of the chronic MPDs suggest that they represent disorders of hematopoietic cell signaling. First, overexpression of numerous growth factor signaling mediators leads to chronic MPDs in mice - for example, unregulated expression of c-Mpl (91) or thrombopoietin (92), loss of either of the signal-inhibitory phosphatases SHP1 (93) and SHIP1 (94), conditional loss of the Ras regulator NF-1 (95), or expression of an activated form of K-Ras (96). Second, hematopoietic cells from patients with chronic MPDs express constitutively activated signaling molecules, including STAT3 (97), Bcl- $x_{\mathrm{L}}$ (98), and Akt (99). Third, hematopoietic progenitor cells are hypersensitive to several different hematopoietic growth factors in PV, IMF, and ET (100-102), including erythropoietin and thrombopoietin; this implies a resetting of the molecular pathways that transduce growth factor signals. Based on these and other observations, several groups hypothesized that abnormalities in JAK2 kinase might underlie the chronic MPDs, and multiple groups reported this to be the case; $65-97 \%$ of patients with PV, $35-57 \%$ of patients with IMF, and $23-57 \%$ of patients with ET carry 1 or 2 mutant JAK2 alleles (overall, two-thirds are heterozygous and one-third homozygous, the latter because of mitotic recombination). Remarkably, every patient studied displays the same acquired mutation, Val-to-Phe substitution of amino acid 617, which leads to constitutive JAK2 activation in vitro, and polycythemia when introduced into hematopoietic cells in vivo (102-107). This region of the pseudokinase domain of JAK2 is necessary for proper JAK2 regulation (65) and, in a molecular model, interacts with the activation loop of the JH1 kinase domain of the molecule (Figure 4) (66). While of great interest, the finding of an activated signaling kinase in patients with chronic myeloproliferative diseases has also raised several questions: why is overexpression of the kinase necessary to produce cytokine hypersensitivity in vitro and polycythemia in vivo (since levels equivalent to wild-type JAK2 do not suffice); why are 3 distinct clinical disorders associated with the same mutation; are second "hits" necessary to generate disease; and what, if any, hematopoietic cytokine receptor does the mutant JAK2 kinase interact with to produce its effects? Regardless of these questions, it is almost certain that this discovery in chronic myeloproliferative diseases will yield new insights in patients with PV, IMF, and ET and spur research into identifying a therapeutic agent that can inhibit the mutant, but not the wild-type, form of JAK2 kinase.

\section{Future research directions}

Our understanding of the molecular basis of thrombopoiesis has progressed substantially in the past 100 years, beginning with James Homer Wright, who in 1906 provided evidence that megakaryocytes give rise to blood platelets (108). Until this time, little attention had been paid to platelets, then referred to as the "dust of the blood." But despite great progress, many questions remain: What is the mechanistic basis for proplatelet formation, a massive cytoplasmic reorganization of actin and tubulin that, upon fragmentation, generates platelets, and what, if any, are the exogenous signals that trigger the process? What is the reason for megakaryocyte polyploidy, and what are the mechanisms by which these cells uncouple DNA synthesis and cell division, one of the most closely guarded links in cell physiology? What is being sensed in marrow stromal cells that alter their production of thrombopoietin in thrombocytopenia, and what are the signals that accomplish this? How can an alteration in the JAK2 kinase lead to PV in some patients and ET in others? And how are stem cell decisions of lineage commitment orchestrated? Research in thrombopoiesis is presently in a logarithmic growth phase. The years to come will provide many new insights into how platelets develop and how that process is regulated.

Address correspondence to: Kenneth Kaushansky, Department of Medicine, Division of Hematology/Oncology, University of California, San Diego, 402 Dickinson Street, Suite 380, San Diego, California 92103-8811, USA. Phone: (619) 543-2259; Fax: (619) 543-3931; E-mail: kkaushansky@ucsd.edu.
1. Thaulow, E., Erikssen, J., Sandvik, L., Stormorken, H., and Cohn, P.F. 1991. Blood platelet count and function are related to total and cardiovascular death in apparently healthy men. Circulation. 84:613-617.

2. Gupta, G.P., and Massague, J. 2004. Platelets and metastasis revisited: a novel fatty link. J. Clin. Invest. 114:1691-1693. doi:10.1172/JCI200423823.

3. Kato, T., et al. 1998. Native thrombopoietin: structure and function. Stem Cells. 16:322-328.

4. Jagerschmidt, A., et al. 1998. Human thrombopoietin structure-function relationships: identification of functionally important residues. Biochem. J. 333:729-734.

5. Kelemen, E., Cserhati, I., and Tanos, B. 1958. Demonstration and some properties of human thrombopoietin in thrombocythemic sera. Acta. Haematol. 20:350-355

6. McDonald, T.P. 1976. A comparison of platelet size, platelet count, and platelet $35 \mathrm{~S}$ incorporation as assays for thrombopoietin. Br. J. Haematol. 34:257-267.

7. Williams, N. 1991. Is thrombopoietin interleukin 6? Exp. Hematol. 19:714-718.
8. Wendling, F., Varlet, P., Charon, M., and Tambourin, P. 1986. MPLV: a retrovirus complex inducing an acute myeloproliferative leukemic disorder in adult mice. Virology. 149:242-246.

9. Souyri, M., Vigon, I., Penciolelli, J.-F., Tambourin, P., and Wendling, F. 1990. A putative truncated cytokine receptor gene transduced by the myeloproliferative leukemia virus immortalizes hematopoietic progenitors. Cell. 63:1137-1147.

10. Vigon, I., et al. 1992. Molecular cloning and characterization of $M P L$, the human homolog of the $\mathrm{v}$-mpl oncogene: identification of a member of the hematopoietic growth factor receptor superfamily. Proc. Natl. Acad. Sci. U. S. A. 89:5640-5644.

11. Kaushansky, K. 1995. Thrombopoietin: the primary regulator of platelet production. Blood. 86:419-431.

12. Kaushansky, K. 1998. Thrombopoietin. N. Engl. J. Med. 339:746-754.

13. Shinjo, K., et al. 1998. Serum thrombopoietin levels in patients correlate inversely with platelet counts during chemotherapy-induced thrombocytopenia. Lenkemia. 12:295-300.

14. Engel, C., Loeffler, M., Franke, H., and Schmitz, S. 1999. Endogenous thrombopoietin serum levels during multicycle chemotherapy. Br. J. Haematol. 105:832-838.

15. Kosugi, S., et al. 1996. Circulating thrombopoietin level in chronic immune thrombocytopenic purpura. Br. J. Haematol. 93:704-706.

16. Ichikawa, N., et al. 1996. Regulation of serum thrombopoietin levels by platelets and megakaryocytes in patients with aplastic anaemia and idiopathic thrombocytopenic purpura. Thromb. Haemost. 76:156-160.

17. Nagasawa, T., et al. 1998. Serum thrombopoietin level is mainly regulated by megakaryocyte mass rather than platelet mass in human subjects. $\mathrm{Br}$. J. Haematol. 101:242-244.

18. Cerutti, A., Custodi, P., Duranti, M., Noris, P., and Balduini, C.L. 1997. Thrombopoietin levels in patients with primary and reactive thrombocytosis. Br. J. Haematol. 99:281-284.

19. Hsu, H.C., et al. 1999. Circulating levels of thrombopoietic and inflammatory cytokines in patients with clonal and reactive thrombocytosis. J. Lab. Clin. Med. 134:392-397.

20. Griesshammer, M., et al. 1999. Aetiology and clinical significance of thrombocytosis: analysis of 732 
patients with an elevated platelet count. J. Intern. Med. 245:295-300.

21. Wolber, E.M., Fandrey, J., Frackowski, U., and Jelkmann, W. 2001. Hepatic thrombopoietin mRNA is increased in acute inflammation. Thromb. Haemost. 86:1421-1424.

22. Bartocci, A., et al. 1987. Macrophages specifically regulate the concentration of their own growth factor in the circulation. Proc. Natl. Acad. Sci. U. S. A 84:6179-6183.

23. Kuter, D.J., and Rosenberg, R.D. 1995. The reciprocal relationship of thrombopoietin (c-Mpl ligand) to changes in the platelet mass during busulfaninduced thrombocytopenia in the rabbit. Blood. 85:2720-2730.

24. Methia, N., Louache, F., Vainchenker, W., and Wendling, F. 1993. Oligodeoxynucleotides antisense to the proto-oncogene c-mpl specifically inhibit in vitro megakaryocytopoiesis. Blood. 82:1395-1401.

25. Cardier, J.E., and Dempsey, J. 1998. Thrombopoietin and its receptor, $\mathrm{c}-\mathrm{mpl}$, are constitutively expressed by mouse liver endothelial cells: evidence of thrombopoietin as a growth factor for liver endothelial cells. Blood. 91:923-929.

26. Geddis, A.E., Fox, N.E., and Kaushansky, K. The $\mathrm{Mpl}$ receptor expressed on endothelial cells does not contribute significantly to the regulation of circulating thrombopoietin (TPO) levels. Exp. Hematol. In press.

27. Sungaran, R., Markovic, B., and Chong, B.H. 1997. Localization and regulation of thrombopoietin mRNA expression in human kidney, liver, bone marrow and spleen using in situ hybridization. Blood. 89:101-107.

28. Sungaran, R., et al. 2000. The role of platelet alphagranular proteins in the regulation of thrombopoietin messenger RNA expression in human bone marrow stromal cells. Blood. 95:3094-3101.

29. Yamashita, K., et al. 2000. Hepatocyte growth factor/scatter factor enhances the thrombopoietin mRNA expression in rat hepatocytes and cirrhotic rat livers. J. Gastroenterol. Hepatol. 15:83-90.

30. Kaser, A., et al. 2001. Interleukin-6 stimulates thrombopoiesis through thrombopoietin: role in inflammatory thrombocytosis. Blood. 98:2720-2725.

31. Gainsford, T., et al. 1998. Cytokine production and function in c-mpl-deficient mice: no physiologic role for interleukin-3 in residual megakaryocyte and platelet production. Blood. 91:2745-2752.

32. Chen, Q., Solar, G., Eaton, D.L., and de Sauvage, F.J. 1998. IL-3 does not contribute to platelet production in c-Mpl-deficient mice. Stem Cells. 16(Suppl. 2):31-36

33. Gainsford, T., et al. 2000. The residual megakaryocyte and platelet production in c-mpl-deficient mice is not dependent on the actions of interleukin-6, interleukin-11, or leukemia inhibitory factor. Blood. 95:528-534.

34. Hodohara, K., Fujii, N., Yamamoto, N., and Kaushansky, K. 2000. Stromal cell derived factor 1 acts synergistically with thrombopoietin to enhance the development of megakaryocytic progenitor cells. Blood. 95:769-775.

35. Hamada, T., et al. 1998. Transendothelial migration of megakaryocytes in response to stromal cellderived factor 1 (SDF-1) enhances platelet formation. J. Exp. Med. 188:539-548.

36. Fox, N.E., and Kaushansky, K. 2005. Engagement of integrin $\alpha 4 \beta 1$ enhances thrombopoietin induced megakaryopoiesis. Exp. Hematol. 33:94-99.

37. Avecilla, S.T., et al. 2004. Chemokine-mediated interaction of hematopoietic progenitors with the bone marrow vascular niche is required for thrombopoiesis. Nat. Med. 10:64-71.

38. Broudy, V.C., Lin, N.L., and Kaushansky, K. 1995. Thrombopoietin (c-mpl ligand) acts synergistically with erythropoietin, stem cell factor, and IL-11 to enhance murine megakaryocyte colony growth and increases megakaryocyte ploidy in vitro. Blood. 85:1719-1726

39. Kaushansky, K., et al. 1995. Thrombopoietin, the Mpl-ligand, is essential for full megakaryocyte development. Proc. Natl. Acad. Sci. U. S. A. 92:3234-3238.

40. Chen, J., Herceg-Harjacek, L., Groopman, J.E., and Grabarek, J. 1995. Regulation of platelet activation in vitro by the c-Mpl ligand, thrombopoietin. Blood. 86:4054-4062.

41. Oda, A., et al. 1996. Thrombopoietin primes human platelet aggregation induced by shear stress and multiple agonists. Blood. 87:4664-4670.

42. Van Os, E., et al. 2003. Thrombopoietin increases platelet adhesion under flow and decreases rolling. Br. J. Haematol. 121:482-490.

43. Sitnicka, E., et al. 1996. The effect of thrombopoietin on the proliferation and differentiation of murine hematopoietic stem cells. Blood. 87:4998-5005.

44. Kobayashi, M., Laver, J.H., Kato, T., Miyazaki, H., and Ogawa, M. 1996. Thrombopoietin supports proliferation of human primitive hematopoietic cells in synergy with steel factor and/or interleukin-3. Blood. 88:429-436.

45. Carver-Moore, K., et al. 1996. Low levels of erythroid and myeloid progenitors in thrombopoietin- and c-mpl-deficient mice. Blood. 88:803-808.

46. Ballmaier, M., et al. 2001. c-mpl mutations are the cause of congenital amegakaryocytic thrombocytopenia. Blood. 97:139-146.

47. Solar, G.P., et al. 1998. Role of c-mpl in early hematopoiesis. Blood. 92:4-10.

48. Kimura, S., Roberts, A.W., Metcalf, D., and Alexander, W.S. 1998. Hematopoietic stem cell deficiencies in mice lacking c-Mpl, the receptor for thrombopoietin. Proc. Natl. Acad. Sci. U. S. A. 95:1195-1200.

49. Zhang, P., et al. 1999. Negative cross-talk between hematopoietic regulators: GATA proteins repress PU.1. Proc. Natl. Acad. Sci. U. S. A. 96:8705-8710.

50. Nerlov, C., Querfurth, E., Kulessa, H., and Graf, T. 2000. GATA-1 interacts with the myeloid PU.1 transcription factor and represses PU.1-dependent transcription. Blood. 95:2543-2551.

51. Emambokus, N., et al. 2003. Progression through key stages of haematopoiesis is dependent on distinct threshold levels of c-Myb. EMBO J. 22:4478-4488

52. Metcalf, D., et al. 2005. Anomalous megakaryocytopoiesis in mice with mutations in the $\mathrm{c}-\mathrm{Myb}$ gene. Blood. 105:3480-3487.

53. Schulze, H., and Shivdasani, R.A. 2004. Molecular mechanisms of megakaryocyte differentiation. Semin. Thromb. Hemost. 30:389-398.

54. Ihle, J.N. 1995. Cytokine receptor signaling. Nature. 377:591-594.

55. Alexander, W.S., and Dunn, A.R. 1995. Structure and transcription of the genomic locus encoding murine c-Mpl, a receptor for thrombopoietin. Oncogene. 10:795-803.

56. Li, J., Sabath, D.F., and Kuter, D.J. 2000. Cloning and functional characterization of a novel c-mpl variant expressed in human CD34 cells and platelets. Cytokine. 12:835-844

57. Coers, J., Ranft, C., and Skoda, R.C. 2004. A truncated isoform of c-Mpl with an essential C-terminal peptide targets the full-length receptor for degradation. J. Biol. Chem. 279:36397-36404.

58. Millot, G.A., et al. 2002. MplK, a natural variant of the thrombopoietin receptor with a truncated cytoplasmic domain, binds thrombopoietin but does not interfere with thrombopoietin-mediated cell growth. Exp. Hematol. 30:166-175.

59. Broudy, V.C., Lin, N.L., Sabath, D.F., Papayannopoulou, T., and Kaushansky, K. 1997. Human platelets display high affinity receptors for thrombopoietin. Blood. 89:1896-1904.

60. Li, J., Xia, Y., and Kuter, D.J. 1999. Interaction of thrombopoietin with the platelet $\mathrm{c}-\mathrm{mpl}$ receptor in plasma: binding, internalization, stability and pharmacokinetics. Br. J. Haematol. 106:345-356.

61. Moliterno, A.R., Hankins, W.D., and Spivak, J.L. 1998. Impaired expression of the thrombopoietin receptor by platelets from patients with polycythemia vera. N. Engl. J. Med. 338:572-580.

62. Yoon, S.Y., Li, C.Y., and Tefferi, A. 2000. Megakaryocyte c-Mpl expression in chronic myeloproliferative disorders and the myelodysplastic syndrome: immunoperoxidase staining patterns and clinical correlates. Eur. J. Haematol. 65:170-174.

63. Huang, L.J., Constantinescu, S.N., and Lodish, H.F. 2001. The N-terminal domain of Janus kinase 2 is required for Golgi processing and cell surface expression of erythropoietin receptor. Mol. Cell. 8:1327-1338.

64. Livnah, O., et al. 1999. Crystallographic evidence for preformed dimers of erythropoietin receptor before ligand activation. Science. 283:987-993.

65. Saharinen, P., Vihinen, M., and Silvennoinen, O. 2003. Autoinhibition of Jak2 tyrosine kinase is dependent on specific regions in its pseudokinase domain. Mol. Biol. Cell. 14:1448-1459.

66. Lindauer, K., Loerting, T., Liedl, K.R., and Kroemer, R.T. 2001. Prediction of the structure of human Janus kinase 2 (JAK2) comprising the two carboxyterminal domains reveals a mechanism for autoregulation. Protein Eng. 14:27-37.

67. Drachman, J.G., Millett, K.M., and Kaushansky, K. 1999. Mpl signal transduction requires functional JAK2, not TYK2. J. Biol. Chem. 274:13480-13484.

68. Miyakawa, Y., Rojnuckarin, P., Habib, T., and Kaushansky, K. 2001. Thrombopoietin induces PI3K and SHP2 activation through Gab and IRS proteins in BaF3 cells and primary murine megakaryocytes. J. Biol. Chem. 276:2494-2502.

69. Bouscary, D., et al. 2001. Role of Gab proteins in phosphatidylinositol 3-kinase activation by thrombopoietin (Tpo). Oncogene. 20:2197-2204.

70. Geddis, A., Fox, N., and Kaushansky, K. 2001. Phosphatidylinositol 3-kinase (PI3K) is necessary but not sufficient for thrombopoietin-induced proliferation in engineered $\mathrm{Mpl}$-bearing cell lines as well as in primary megakaryocytic progenitors. J. Biol. Chem. 276:34473-34479.

71. Kojima, H., et al. 2001. Role of phosphatidylinositol-3 kinase and its association with Gab1 in thrombopoietin-mediated up-regulation of platelet function. Exp. Hematol. 29:616-622.

72. Drachman, J.D., Sabath, D.F., Fox, N.E., and Kaushansky, K. 1997. Thrombopoietin signal transduction in purified murine megakaryocytes. Blood. 89:483-492.

73. Kirito, K., Fox, N.E., and Kaushansky, K. 2003. Thrombopoietin stimulates expression of HoxB4: an explanation for the favorable effects of TPO on hematopoietic stem cells. Blood. 102:3172-3178.

74. Rojnuckarin, P., et al. 2001. The roles of PI3K and PKC $\xi$ for thrombopoietin-induced MAP kinase activation in primary murine megakaryocytes. J. Biol. Chem. 276:41014-41022.

75. Kirito, K., Fox, N.E., and Kaushansky, K. 2004. Thrombopoietin (TPO) induces the nuclear translocation of HoxA9 in hematopoietic stem cells (HSC): a potential explanation for the favorable effects of TPO on HSCs. Mol. Cell. Biol. 24:6751-6762.

76. Rojnuckarin, P., Drachman, J.G., and Kaushansky, K. 1999. Thrombopoietin-induced activation of the mitogen activated protein kinase pathway in normal megakaryocytes: role in endomitosis. Blood. 94:1273-1282.

77. van Willigen, G., Gorter, G., and Akkerman, J.W 2000. Thrombopoietin increases platelet sensitivity to alpha-thrombin via activation of the ERK2cPLA2 pathway. Thromb. Haemost. 83:610-616.

78. Ishibashi, T., et al. 1989. Human interleukin 6 is a direct promoter of maturation of megakaryocytes in vitro. Proc. Natl. Acad. Sci. U. S. A. 86:5953-5957.

79. Laterveer, L., van Damme, J., Willemze, R., and 
Fibbe, W.E. 1993. Continuous infusion of interleukin- 6 in sublethally irradiated mice accelerates platelet reconstitution and the recovery of myeloid but not of megakaryocytic progenitor cells in bone marrow. Exp. Hematol. 21:1621-1627.

80. Sosman, J.A., et al. 1997. Concurrent phase I trials of intravenous interleukin 6 in solid tumor patients: reversible dose-limiting neurological toxicity. Clin. Cancer Res. 3:39-46.

81. Kozak, M. 2001. Constraints on reinitiation of translation in mammals. Nucleic Acids Res. 29:5226-5232.

82. Cazzola, M., and Skoda, R.C. 2000. Translational pathophysiology: a novel molecular mechanism of human disease. Blood. 95:3280-3288.

83. Ihara, K., et al. 1999. Identification of mutations in the c-mpl gene in congenital amegakaryocytic thrombocytopenia. Proc. Natl. Acad. Sci. U. S. A. 96:3132-3136

84. van den Oudenrijn, S., et al. 2000. Mutations in the thrombopoietin receptor, $\mathrm{Mpl}$, in children with congenital amegakaryocytic thrombocytopenia. Br. J. Haematol. 110:441-448.

85. Ballmaier, M., Germeshausen, M., Krukemeier, S., and Welte, K. 2003. Thrombopoietin is essential for the maintenance of normal hematopoiesis in humans: development of aplastic anemia in patients with congenital amegakaryocytic thrombocytopenia. Ann. N. Y. Acad. Sci. 996:17-25.

86. Sabath, D.F., Kaushansky, K., and Broudy, V.C. 1999. Deletion of the membrane-distal cytokine receptor homology domain of MPL results in constitutive cell growth and loss of thrombopoietin binding. Blood. 94:365-367.

87. Onishi, M., et al. 1996. Identification of an oncogenic form of the thrombopoietin receptor using retrovirus-mediated gene transfer. Blood. 88:1399-1406.

88. Ding, J., et al. 2004. Familial essential thrombocythemia associated with a dominant-positive activating mutation of the c-MPL gene, which encodes for the receptor for thrombopoietin. Blood. 103:4198-4200.

89. Moliterno, A.R., et al. 2004. Mpl Baltimore: a thrombopoietin receptor polymorphism associated with thrombocytosis. Proc. Natl. Acad. Sci. U. S. A. 101:11444-11447.

90. Spivak, J.L., et al. 2003. Chronic myeloproliferative disorders. Hematology (Am. Soc. Hematol. Educ. Program). 2003:200-224.

91. Cocault, L., et al. 1996. Ectopic expression of murine TPO receptor $(\mathrm{c}-\mathrm{mpl})$ in mice is pathogenic and induces erythroblastic proliferation. Blood. 88:1656-1665.

92. Yan, X.-Q., et al. 1996. A model of myelofibrosis and osteosclerosis in mice induced by overexpressing thrombopoietin (mpl ligand): reversal of disease by bone marrow transplant. Blood. 88:402-409.

93. Tsui, H.W., Siminovitch, K.A., de Souza, L., and Tsui, F.W. 1993. Motheaten and viable motheaten mice have mutations in the haematopoietic cell phosphatase gene. Nat. Genet. 4:124-129.

94. Helgason, C.D., et al. 1998. Targeted disruption of SHIP leads to hemopoietic perturbations, lung pathology, and a shortened life span. Genes Dev. 12:1610-1620.

95. Gitler, A.D., et al. 2004. Tie2-Cre-induced inactivation of a conditional mutant Nf1 allele in mouse results in a myeloproliferative disorder that models juvenile myelomonocytic leukemia. Pediatr. Res. 55:581-584.

96. Chan, I.T., et al. 2004. Conditional expression of oncogenic K-ras from its endogenous promoter induces a myeloproliferative disease. J. Clin. Invest. 113:528-538. doi:10.1172/JCI200420476.

97. Roder, S., Steimle, C., Meinhardt, G., and Pahl, H.L. 2001. STAT3 is constitutively active in some patients with Polycythemia rubra vera. Exp. Hematol. 29:694-702.

98. Silva, M., et al. 1998. Expression of Bcl-x in ery- throid precursors from patients with polycythemia vera. N. Engl. J. Med. 338:564-571.

99. Dai, C., Chung, I.J., and Krantz, S.B. 2005. Increased erythropoiesis in polycythemia vera is associated with increased erythroid progenitor proliferation and increased phosphorylation of Akt/PKB. Exp. Hematol. 33:152-158.

100. Zanjani, E.D., Lutton, J.D., Hoffman, R., and Wasserman, L.R. 1997. Erythroid colony formation by polycythemia vera bone marrow in vitro. Dependence on erythropoietin. J. Clin. Invest. 59:841-848.

101.Dai, C.H., et al. 1992. Polycythemia vera. II. Hypersensitivity of bone marrow erythroid, granulocytemacrophage, and megakaryocyte progenitor cells to interleukin-3 and granulocyte-macrophage colony-stimulating factor. Blood. 80:891-899.

102.Axelrad, A.A., Eskinazi, D., Correa, P.N., and Amato, D. 2000. Hypersensitivity of circulating progenitor cells to megakaryocyte growth and development factor (PEG-rHu MGDF) in essential thrombocythemia. Blood. 96:3310-3321.

103.Baxter, E.J., et al. 2005. Acquired mutation of the tyrosine kinase JAK2 in human myeloproliferative diseases. Lancet. 365:1054-1061.

104.Levine, R.L., et al. 2005. Activating mutation in the tyrosine kinase JAK2 in polycythemia vera, essential thrombocythemia, and myeloid metaplasia with myelofibrosis. Cancer Cell. 7:387-397.

105.James, C., et al. 2005. A unique clonal JAK2 mutation leading to constitutive signalling causes polycythaemia vera. Nature. 434:1144-1148.

106. Kralovics, R., et al. 2005. A gain-of-function mutation of JAK2 in myeloproliferative disorders. N. Engl. J. Med. 352:1779-1790.

107.Zhao, R., et al. 2005. Identification of an acquired JAK2 mutation in polycythemia vera. J. Biol. Chem. 280:22788-22792.

108. Wright, J.H. 1906. The origin and nature of the blood plates. Boston Med. Surg. J. 23:643-645. 